\title{
MicroRNA-125a-3p is involved in early behavioral disorders in stroke-afflicted rats through the regulation of Cadm2
}

\author{
YUQING LIU ${ }^{1}$, YUNJUN LI ${ }^{2}$, ZHENXING REN $^{1}$, WENWEN SI ${ }^{1}$, YIWEI LI $^{3}$, GANG WEI $^{4}$, \\ WENGUANG ZHAO ${ }^{5}$, JIANHONG ZHOU ${ }^{1}$, YAGE TIAN ${ }^{1}$ and DONGFENG CHEN ${ }^{1}$ \\ ${ }^{1}$ Department of Anatomy, The Research Center of Integrative Medicine, Guangzhou University of \\ Traditional Chinese Medicine, Guangzhou, Guangdong 510006; ${ }^{2}$ Center of Sanxi Community Health Service, \\ Shenzhen Dapeng District Maternal and Child Health Care Hospital, Shenzhen, Guangdong 518120; \\ ${ }^{3}$ School of Nursing; ${ }^{4}$ Research and Development of New Drugs; ${ }^{5}$ School of Medical Information Engineering, \\ Guangzhou University of Traditional Chinese Medicine, Guangzhou, Guangdong 510006, P.R. China
}

Received February 17, 2017; Accepted October 6, 2017

DOI: $10.3892 / \mathrm{ijmm} .2017 .3179$

\begin{abstract}
Ischemic strokes carry a significant risk of mortality and recurrent vascular events. Recent studies suggest that changes in microRNAs (miRNAs or miRs) may affect the development of the stroke. However, few studies have investigated the role of miRNAs in behavioral disorder in early stroke. In the present study, animal models of middle cerebral artery occlusion (MCAO) are used, as well as a cell model of neurite outgrowth to further investigate the role of miRNAs in targeting synapse-associated proteins expression in early stroke. The authors used miRNA expression microarrays on RNA extracted from the cortex tissue samples from the rats of MCAO and control rats. Reverse transcription-quantitative polymerase chain reaction was conducted to verify the candidate miRNAs discovered by microarray analysis. Data indicated that miR-125a was significantly increased in the cortex of the model of MCAO, which were concomitant with that rats of MCAO at the same age displayed significant behavioral deficits. Bioinformatics analysis predicted the cell adhesion molecule 2 (Cadm2, mRNA) neurite outgrowthassociated protein is targeted by $\mathrm{miR}-125 \mathrm{a}$. Overexpression of miR-125a reduced the level of Cadm2 expression in PC12 cell injury induced by free-serum. In contrast, inhibition of miR-125a using miR-125a inhibitors significantly resulted in higher levels of Cadm2 expression. In conclusion, miR-125a is involved in the behavioral disorder of animal models of MCAO by regulation of Cadm2.
\end{abstract}

Correspondence to: Professor Dongfeng Chen, Department of Anatomy, The Research Center of Integrative Medicine, Guangzhou University of Traditional Chinese Medicine, Guangzhou, Guangdong 510006, P.R. China

E-mail: cdf27212@21cn.com

Key words: microRNA-125a, cell adhesion molecule 2, stroke, neurite outgrowth, behavioral disorder

\section{Introduction}

Stroke is complex neurological diseases in pathophysiology with high morbidity and is commonly associated with multiple and long standing impairments $(1,2)$. Ischemic stroke should be taken seriously in the early stage of pathological process for study. It is significant to seek for therapeutic targets for effective protection of cerebral ischemic impairments $(3,4)$. Extensive research has indicated that ischemic stroke is followed by highly complex mechanisms such as the inflammatory (5-7) response, and autophagy (8) and apoptosis (9-11), leading to ischemic neuronal ischemic and necrotic death, resulting in cognitive function (12) and behavior and other impairments $(13,14)$. The mechanism of stroke-induced cognitive impairment has been extensively studied, while behavioral disorders caused by cerebral ischemic stroke have been poorly studied. Neural cell adhesion molecule 2 (Cadm2), which is a synapse-associated protein, is a cell adhesion factor that has critical roles in the regulation of neuronal motility and neurite elongation in developing neurons $(15,16)$. The changes in expression of $\mathrm{Cadm} 2$ have been proposed to contribute to nerve growth disorders in humans (17). However, the role of Cadm 2 in the early stage of stroke remains poorly understood. Therefore, the extensive identification of a possible basis for synapse-associated proteins may provide a new strategy for the treatment of stroke disorders.

Previous studies have revealed that aberrant regulation of microRNA (miRNA or miR) expression has been linked with the onset and progression of stroke $(18,19)$. miRNAs are noncoding RNA molecules that regulate gene expression through binding 3'-untranslated region (3'-UTR) site in the mRNA to repress translation or induce mRNA cleavage (20). To data, miRNAs has been extensively studied and has been shown to serve an important role in the process of cell growth $(21,22)$, proliferation $(23,24)$, differentiation $(25,26)$, development $(27,28)$ and apoptosis $(29,30)$. It has been reported that miRNAs are involved in the pathophysiology of ischemic stroke by regulating their target genes (31). Moreover, miRNAs play important roles in neurite extension and branching $(32,33)$. However, limited research is available on the correlation of 
miRNAs with synapse-associated proteins, particularly during the early stages of ischemic stroke. In the present study, the authors used the animal models of middle cerebral artery occlusion (MCAO) and cell model of neurite outgrowth to further investigate the role of miRNAs in targeting synapseassociated proteins, expression involved in behavioral disorder during the early stage of ischemic stroke. In the present study, the authors used the animal models of MCAO and cell model of neurite outgrowth to further investigate the role of miRNAs in targeting synapse-associated proteins expression involved in behavioral disorder during the early stage of ischemic stroke.

\section{Materials and methods}

Animals. Sprague-Dawley (SD) rats were obtained from the Department of Animal Experiments, Guangzhou University of Chinese Medicine. Animals used in all experimental procedures were procured from the Central Animal House Facility Animal Experimental Center, Guangzhou Medicine University (Guangzhou, China), under standard laboratory conditions at a temperature $\left(20-25^{\circ} \mathrm{C}\right), 12 / 12 \mathrm{~h}$ light/dark cycle and were provided with a normal pellet diet, water provided ad libitum. All animals received human care in accordance with the guideline set by the Care of Experiment Animals Committee of Guangzhou University of Chinese Medicine. The study was approved by the Experiment Animals Committee of Guangzhou University of Chinese Medicine (Guangzhou, China).

PC12 cells culture. PC12 cells were purchased from Shanghai Institute of Cell Science, Chinese Academy of Sciences (Shanghai, China), and cultured at $37^{\circ} \mathrm{C}, 5 \% \mathrm{CO}_{2}$ in RPMI-1640 medium, $10 \%$ fetal bovine serum (both from Thermo Fisher Scientific, Inc., Waltham, MA, USA), 1\% penicillin-streptomycin (HyClone; GE Healthcare Life Sciences, Chalfont, UK). The medium was changed every 3 days. For neurite outgrowth experiments, after placing in reduced serum medium, PC12 cells were plated on 24- or 6-well cell culture plates coated with ploy-l-lysine at a density $2 \times 10^{4}$ or $3 \times 10^{5}$ cells/well, respectively.

Induction of cerebral ischemia. Male SD rats (200-230 g) were randomized into two groups ( $n=10 /$ group): Control group (normal) and model group. Model group was induced in adult male SD rats (280-300 g) using the intraluminal filament technique. Rats were anesthetized with an intraperitoneal injection of $10 \%$ chloral hydrate at a dose of $0.33 \mathrm{ml} / 100 \mathrm{~g}$. A midline neck incision was made; the right common carotid artery and external carotid artery were isolated. A nylon filament was inserted into the middle cerebral artery and maintained for $120 \mathrm{~min}$. Reperfusion was achieved by withdrawing the suture after 120 min of occlusion. Following the operation, rats were transferred to a temperature-controlled chamber to maintain body temperature at $37.5^{\circ} \mathrm{C}$. Following $120 \mathrm{~min}$ of occlusion, rats were scored for neurological function. The neurologic scoring method was based on Longa method which was classified as a five-point scale (34): Point 0 , no neurologic deficit; point 1 , failure to extend the left forepaw fully; point 2 , constant circling to the left; point 3 , falling to the left side; and point 4 , no spontaneous walking with a depressed level of consciousness. After neurological evaluation of MCAO rats, brains were removed and placed on ice. Seven brains were removed and fixed in $10 \%$ buffered formalin phosphate for 24 to $48 \mathrm{~h}$ for paraffin embedding. Serial coronal frontal cortex and cortex tissue sections $(5 \mu \mathrm{m})$ were cut and every tenth section was systematically assigned to a series of sections, yielding a total of 10 series. One series of sections was saved for immunohistochemical analysis. For reverse transcriptionquantitative polymerase chain reaction (RT-qPCR) and western blot analysis, six ipsilateral cortices and cortex tissue were rapidly removed and placed on ice. The cortices and cortex tissue were stored at $-80^{\circ} \mathrm{C}$ for analysis.

microRNA microarray assay. Total RNA was extracted from cortex tissues using TRIzol reagent according to manufacturer's instructions (Life Technologies, Carlsbad, CA, USA). The RNA was subsequently fluorescently labeled with cy3 by ULS notation, and hybridized to CustomArray ${ }^{\mathrm{TM}}$ microarray following the manufacture's instructions (Guangzhou RiboBio Co., Ltd., Guangzhou, China). In order to decrease the specific hybridization background, the microarray was washed and the imaging solution was taken to the semiconductor microarray. Data were analyzed by MeV (Multi Experiment Viewer 4.9.0; Springer, Boston, MA, USA).

$R T-q P C R$. Total RNA was extracted using TRIzol reagent (Thermo Fisher Scientific, Inc.) according to the manufacturer's protocol. miR-125a level was analyzed using all-in-one miRNAs RT-qPCR detection kit (GeneCopoeia, Rockville, MD, USA) according to the manufacturer's instructions. The reaction conditions of $95^{\circ} \mathrm{C}$ for $10 \mathrm{sec}, 60^{\circ} \mathrm{C}$ for $20 \mathrm{sec}$ and $72^{\circ} \mathrm{C}$ for $15 \mathrm{sec}$, for a total of 40 cycles. Finally, the relative miR-125a level was normalized to the endogenous control U6 expression for each sample in triplicate and was calculated by the $2^{-\Delta \Delta \mathrm{Ct}}$ method, as previously described (35). The primers used were fluorescent quantitative rno-miR-125a-3p PCR primers, 5'-GACGACAGGTGAGGTTCTTGG-3' (Invitrogen; Thermo Fisher Scientific, Inc.).

Transfection of PC12 cells with miR-125a mimics, inhibitor, miR-125a mimic negative control (mimic NC, $m-N C$ ) and miR-125a inhibitor negative control (inhibitor NC, $i$-NC). $\mathrm{PC} 12$ cells were plated at $3 \times 10^{5}$ cells/well in 6 -well plates 1 day before transfection. Transfected Lipofectamine 2000 (Thermo Fisher Scientific, Inc.) $2.5 \mu \mathrm{l} / \mathrm{ml}$ with $50 \mathrm{nM}$ miR-125a mimics or $100 \mathrm{nM}$ inhibitor for $6 \mathrm{~h}$ according to the instructions of Lipofectamine 2000. Following the terminal transfection for $24 \mathrm{~h}$, the cells were collected with $0.25 \%$ trypsin digestion and lysed with radioimmunoprecipitation assay buffer (Thermo Fisher Scientific, Inc.) for western blot analysis.

Immunofluorescence assay. PC12 cells were plated at $2 \times 10^{4}$ cells/well in 24-well plates 1 day before transfection, then divided into 4 groups: i), control group; ii), model group (free-serum incubation $12 \mathrm{~h}$ ); iii), miR-125a mimic group: transfected miR-125a mimic followed by free-serum incubation for $12 \mathrm{~h}$; iv), miR-125a inhibitor group: transfected miR-125a inhibitor followed by free-serum incubation for 12 h. PC12 cells or tissue sections were incubated with an anti-rabbit Cadm2 antibody (1:200, Ab64873; Abcam, 
Cambridge, UK) overnight in 5\% BSA (Sigma-Aldrich; Merck $\mathrm{KGaA}$, Darmstadt, Germany) at $4^{\circ} \mathrm{C}$. After thorough washing, cells were incubated with a conjugated anti-rabbit secondary antibody (1:500, Alexa Fluor 488, Ab150077; Abcam) for $30 \mathrm{~min}$ at room temperature. Cells were examined under laser confocal (Carl Zeiss AG, Jena, Germany) and analyzed by Image Pro Plus software 6.0 (Media Cybernetics, Inc., Rockville, MD, USA).

Measurement of neurite outgrowth. PC12 cells were inoculated into the wells at the initial density of $2 \times 10^{4}$ cells/well. PC12 cells were transfected with miR-125a mimics, miR-125a inhibitor, miR-125a mimics or inhibitor negative control as immunofluorescence assay described into five groups: i) miR-125a mimics group; ii) miR-125a mimics negative control group (miR-125a mimics NC group); iii) miR-125a inhibitor group; iv) miR-125a inhibitor negative control group (miR-125a inhibitor NC group); v) the control group with normal cultured. For neurite length assessment, the cells were observed under a laser confocal microscope; the 20 longest protrusions in each experimental group were measured and quantified from six independent fields of vision (three experiments per condition). The following indicators were used to observe the effects of miR-125a on cell neurite outgrowth: i) spindle cell rate, the total number of spindle cells/total number of cells, observation of cell body shape; ii) cell differentiation rate D1, the number of cell protrusions/total number of cells; iii) the rate of cell differentiation D2, the number of cells with neurite outgrowth $>2$ cell body lengths/the total number of cells; iv) average protrusion length of cell, the length of the longest protrusions/the number of samples. Neurite length was measured and quantified using the ImageJ 1.4 (National Institutes of Health, Bethesda, MD, USA) and GraphPad Prism 5 (GraphPad Software, Inc., La Jolla, CA, USA) programs.

Western blotting. Briefly, proteins extracted by radioimmunoprecipitation assay buffer (Thermo Fisher Scientific, Inc.) from cultured PC12 cells or tissue followed by 5-10 min boiling and centrifugation at $12,000 \mathrm{xg}$ for $10-15 \mathrm{~min}$ at $4^{\circ} \mathrm{C}$ to obtain the supernatant. Protein concentration was determined with a BCA protein assay kit (Thermo Fisher Scientific, Inc.) according to the manufacturer's instructions. Equal amounts of lysate protein $(20 \mu \mathrm{g} /$ lane $)$ were subjected to $10 \%$ sodium dodecyl sulfate-polyacrylamide gel electrophoresis (SDS-PAGE) and transferred to nitrocellulose membranes (Bio-Rad Laboratories, Inc., Hercules, CA, USA) after electrophoresis. At room temperature, the membranes were blocked with 5\% bovine serum albumin (BSA) in PBST buffer (PBS with $0.01 \%$ Tween-20, $\mathrm{pH} 7.4$ ) and incubated overnight at $4^{\circ} \mathrm{C}$ with antibody of Cadm2 (Ab-64873, dilution, 1:500; Abcam), $\beta$-actin (Bs-0061R, dilution, 1:2,000; Bioss, Beijing, China), in PBST containing $1 \%$ BSA. Immunoreactivity was detected by sequential incubation with horseradish peroxidase-conjugated secondary antibodies donkey anti-rabbit immunoglobulin IgG (cat. no. Ab-6721, dilution, 1:10,000; Abcam) for $2 \mathrm{~h}$ at room temperature, and detected by ECL western blot detection reagent (Bio-Rad Laboratories, Inc.). Densitometry quantitation was used to analyze with ImageJ 1.4 Software (National Institutes of Health).
Co-transfection of the luciferase reporter carrying Cadm2wild-type(WT)3'-UTR mutanttype (MUT), Cadm2-MUT-3'-UTR (WT). Plates (24-well) were plated at a density of $2 \times 10^{4}$ cells/well. PC12 cells were transfected using Lipofectamine 2000(Invitrogen; Thermo Fisher Scientific, Inc.) with Cadm2-WT 3'-UTR, Cadm2-MUT 3'-UTR plasmid and miR-125a mimic, inhibitor, mimic $\mathrm{NC}$ and inhibitor $\mathrm{NC}$ for $6 \mathrm{~h}$ in culture plates. In the cloning vector of psiCHECK2-Cadm2, miRNA target sites were inserted after the Renilla luciferase (hRluc) region, Firefly luciferase (luc) was taken as internal reference. Cells treated with mimic NC, and inhibitor NC served as negative controls. Each value from the wells was collected with the dual luciferase reporter assay system to quantify luminescent signal.

Statistical analysis. Data were reported as mean \pm standard deviation of these independent experiments. Statistical analyses were performed using GraphPad Prism version 5.0 between two groups (GraphPad Software, Inc.). Data were used with test analysis and one-way analysis of variance and $\mathrm{P}<0.05$ was considered to indicate a statistically significant difference.

\section{Results}

Score of neurofunction. To explore whether the mice with MCAO presented early symptoms of behavioral disorder, the authors used Longa method. The present study demonstrated that the MCAO rats showed these symptoms of behavioral disorder, as assessed by the Longa score (Fig. 1B).

miR-125a expression is increased in the cortex of ischemic rats. To determine whether miRNAs exist in the cortex to connect with neurite outgrowth-associated proteins during the development of stroke, the authors used a miRNA microarray assay to detect the association of mRNAs with diseases, such as stroke. Data were analyzed and illustrated with cluster diagram (Fig. 1A). Results showed that the expression of miR-125a in the model group was increased in contrast to the control group, and, considering the acquisition error, the authors also took miR-125a into the following study. RT-qPCR indicated that, compared with the control group, the expression of miR-125a in the cortex of the model group was also higher (Fig. 1C). Moreover, miR-125a may have contact directly with Cadm2, which has the function of affecting neurite outgrowth. In vitro, it is of great significance to the further study of miR-125a.

Cadm2 expression is decreased in the cortex of ischemic rats. To determine whether Cadm2 exists in the cortex to connect with neurite outgrowth-associated proteins in the development of stroke, the authors used an immunofluorescence assay to examine with association with stroke. Data were detected with laser confocal microscope (Fig. 2A). Results indicated that the expression of Cadm2 in the model group was decreased when contrasted to the control group. By using western blotting, compared with the control group, the expression of Cadm2 of the model group was also lower (Fig. 2B).

miR-125a inhibits Cadm2 expression. In previous studies, the authors identified that the neurite outgrowth-associated 

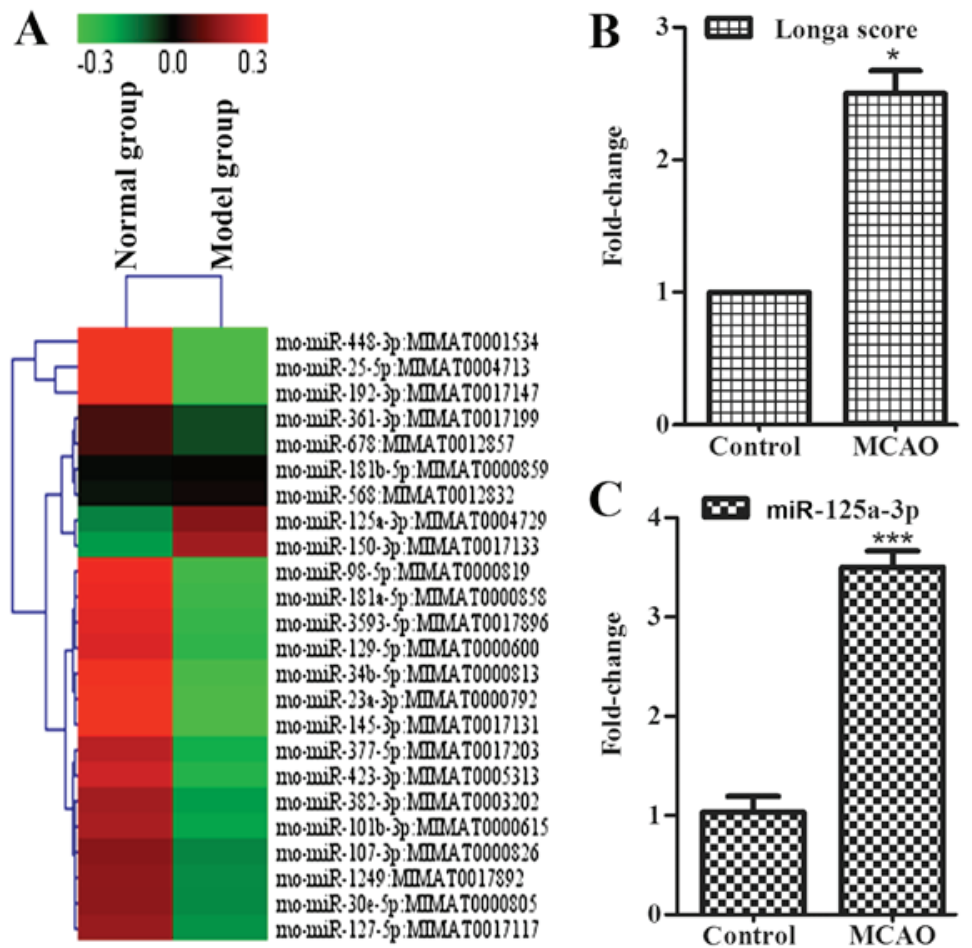

Figure 1. miR-125a was increased in the cortex of ischemic rats. (A) The expression of miRNAs in cortex was measured using microarray analysis in control group (left) and model group (right). (B) Longa score in control group and model group. (C) The expression of miR-125a in cortex were measured using reverse transcription-quantitative polymerase chain reaction in the cortex, data were presented as the mean \pm standard deviation, $n=3$ in each group. "P<0.05 vs. normal group. miR, microRNA; MCAO, middle cerebral artery occlusion; Cadm2, cell adhesion molecule 2.

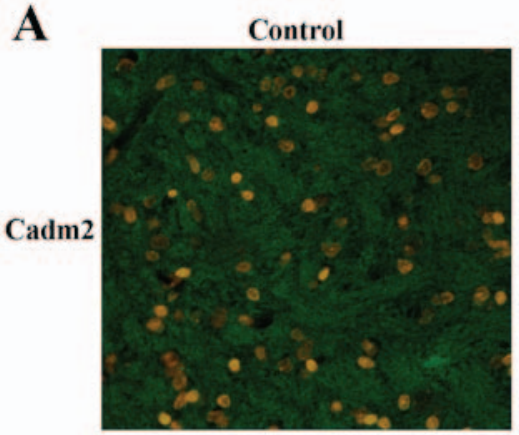

B
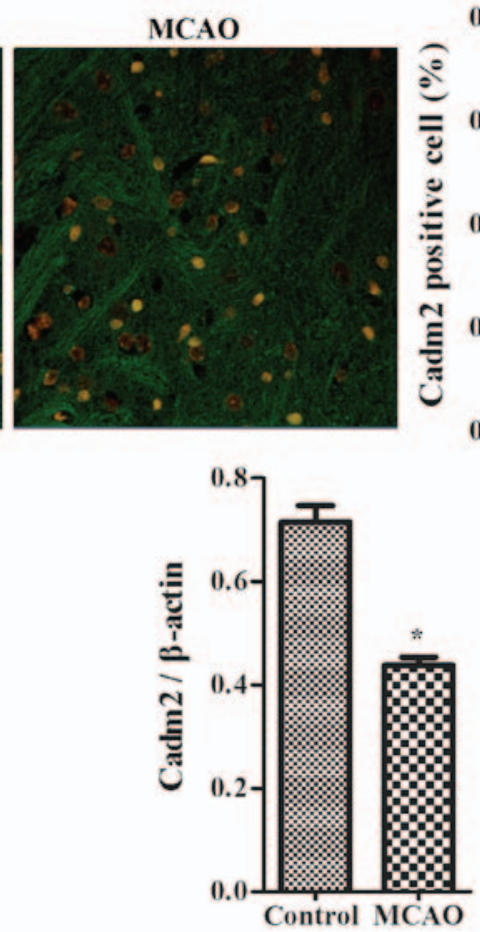
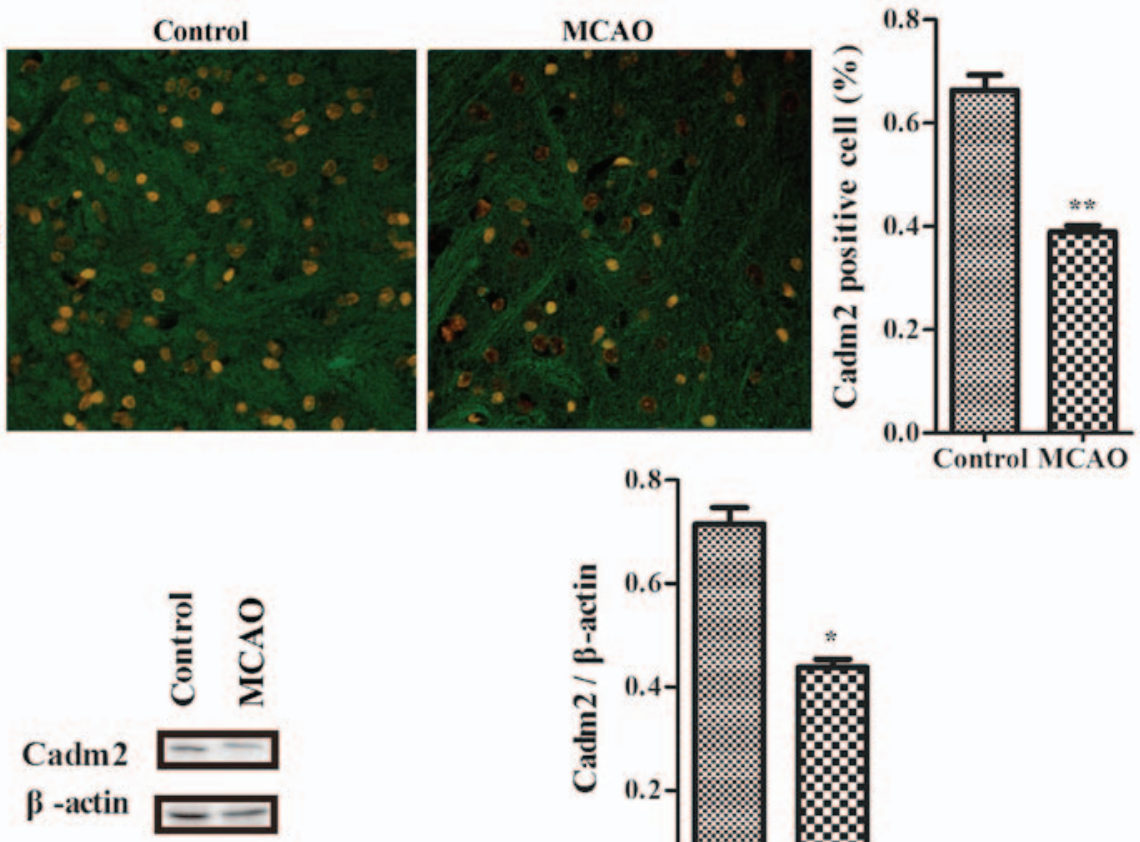

Control MCAO

Figure 2. Cadm2 was decreased in the cortex of ischemic rats. (A) The expression of Cadm2 in cortex was measured by laser confocal microscopy (original magnification, x200). (B) Longa score in control group and model group. The expression of Cadm2 was measured using a western blot assay in the cortex, and the immunoreactive bands were quantified with ImageJ software. By using western blot analysis, the expression of the Cadm2 protein was efficiently decreased in the model group, compared with the control group. ${ }^{*} \mathrm{P}<0.05$ and ${ }^{* *} \mathrm{P}<0.01$ vs. control. MCAO, middle cerebral artery occlusion; Cadm2, cell adhesion molecule 2 .

proteins may be the target protein of miR-125a. For confirming the target protein using PC12 cells in vitro, cells were transfected with miR-125a mimics, miR-125a inhibitor compared with control group and no serum group, PC12 cells 

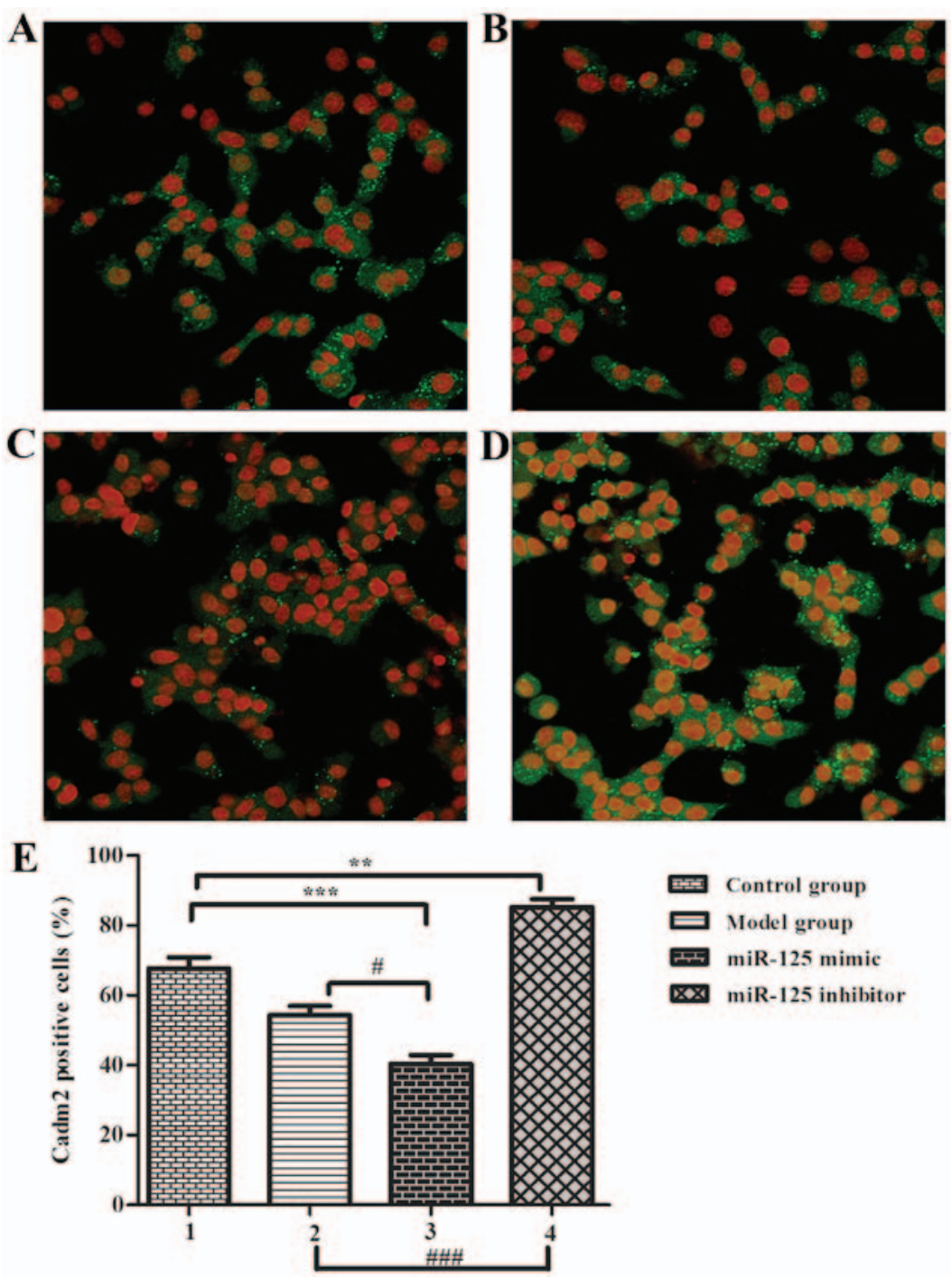

Figure 3. miR-125a inhibited Cadm2 expression (original magnification, x200). (A) Control group, (B) model group (serum-free), (C) miR-125a mimic group, (D) miR-125a inhibitor group. (E) Percentage of Cadm2-positive cells. One-way analysis of variance was used to analyze the differences. ${ }^{* *} \mathrm{P}<0.01$ and

${ }^{* * *} \mathrm{P}<0.001 ;{ }^{\#} \mathrm{P}<0.05$ and ${ }^{\# \#} \mathrm{P}<0.001$ as indicated. miR, microRNA. Cadm2, cell adhesion molecule 2.

were used as a serum-free cell model (12 h) for observing neurite outgrowth, results were observed in an immunofluorescence assay (Fig. 3). Results showed that the ratio of positive cells in the model group (Fig. 3B) was lower than the control group (Fig. 3A). The mimic group (Fig. 3C) positive cell number was lower than the inhibitor group (Fig. 3D) and the control group. These results indicate that serum-free miR-125a could inhibited neurite outgrowth in stroke development process (Fig. 3E), thus, PC12 cells could be suitable for the authors' further study to prove the relationship between Cadm2 and miR-125a.

miR-125a inhibits neurite outgrowth in PC12 cells. To further validate the role of Cadm2 in miR-125a mediated neurite outgrowth, PC12 cells were transfected with miR-125a mimics, inhibitor, mimic NC or inhibitor NC and the neurite length was reported (Fig. 4). The results demonstrated that cells transfected with the miR-125a mimic had a significantly decreased neurite extension compared than miR-125a mimic NC and control groups in terms of spindle cell rate (Fig. 4A), cell differentiation rate D1 (Fig. 4B), the rate of cell differentiation D2 (Fig. 4C) and average protrusion length of cell (Fig. 4D). PC12 cells transfected with miR-125a inhibitor, which suppresses mature miR-125a expression (Fig. 4), exhibited a significant increase in neurite outgrowth.

miR-125a can specifically reduce the protein expression of Cadm2. To investigate whether miR-125a inhibits the expression of target protein Cadm2, the authors transfected cells with miR-125a mimic or inhibitor, or miR-125a mimic NC or inhibitor NC into PC12 cells. It was determined that Cadm2 with the function of neurite outgrowth was a potential target of miR-125a. Compared with the control group and mimic $\mathrm{NC}$, the expression of Cadm 2 protein was reduced in mimic group by western blotting and the results were analyzed with ImageJ (Fig. 5B). The expression of Cadm2 protein was increased in the inhibitor group, contrasted to the control group inhibitor NC. To identify whether miR-125a targeted the expression of $\mathrm{Cadm} 2$, the authors co-transfected the cells with miR-125a mimic, inhibitor or miR-125a mimic NC, inhibitor NC with Cadm2-WT 3'-UTR or Cadm2-MUT 3'-UTR into PC12 cells. Results indicated that the WT+miR-125a mimic 

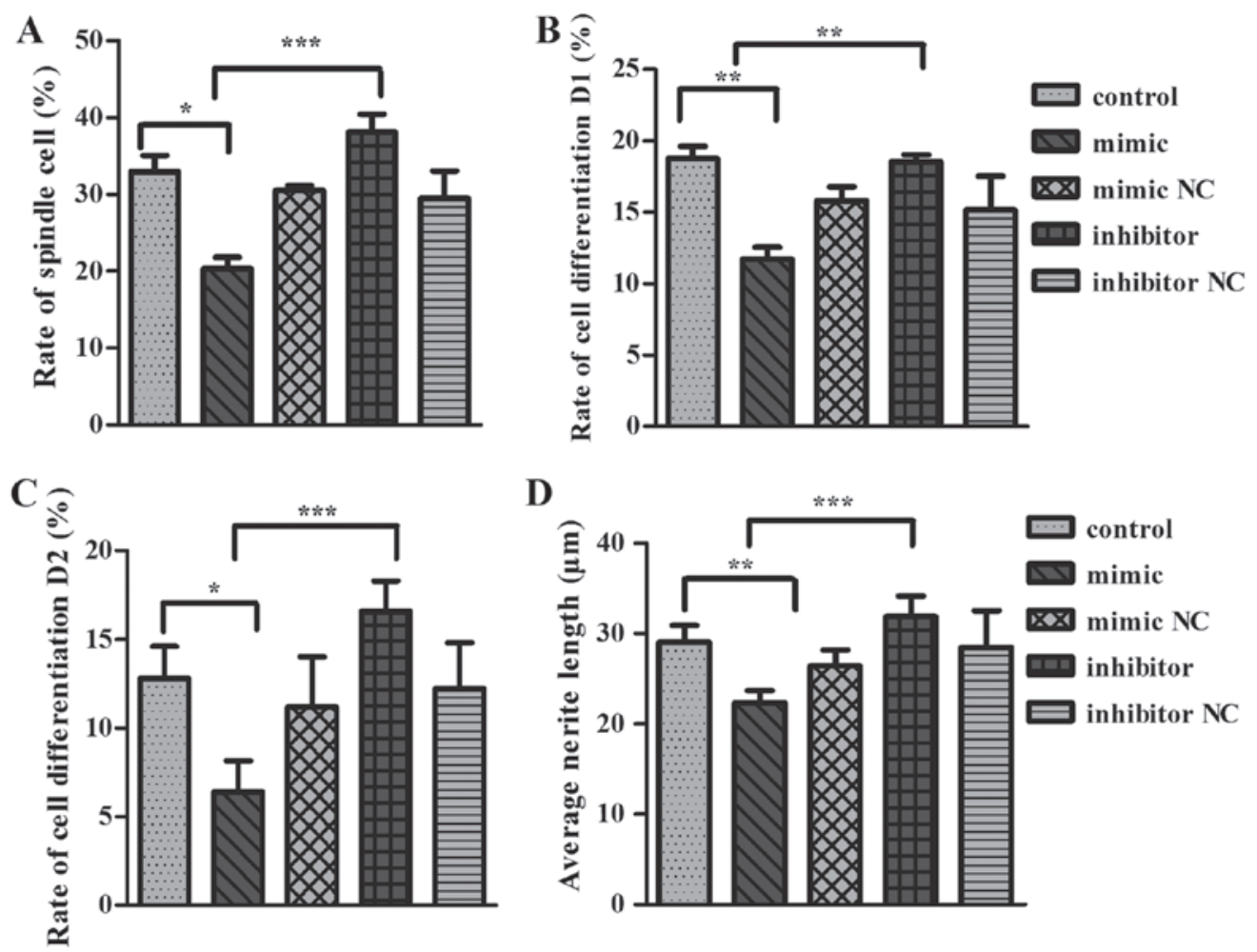

Figure 4. microRNA-125a inhibited neurite outgrowth in PC12 cells. (A) Spindle cell rate, (B) cell differentiation rate D1, (C) cell differentiation rate D2, (D) average protrusion length of cell. One-way analysis of variance test was used to analyze the variances between the groups ${ }^{*} \mathrm{P}<0.05$ and ${ }^{* * * *} \mathrm{P}<0.001$ as indicated. NC, negative control.

significantly reduced the luciferase activity, compared with MUT+miR-125a mimic and mimic NC. The WT+miR-125a inhibitor increased the luciferase activity, compared with MUT+miR-125a inhibitor and inhibitor NC results reported that miR-125a specifically regulated the expression of Cadm2, inhibiting neurite outgrowth (Fig. 5C).

\section{Discussion}

Stoke is the result of ischemic brain and can lead to neuronal damage (36), and there is no effective cure for this condition. Ischemic brain injury to neuronal cells is mediated by multiple mechanisms (37). One of the processes that may play a key role in the delayed progression of the neurite outgrowth is initiated by the expression of miRNAs (38). However, there are few studies on the roles of miRNAs in the behavioral disorder associated with the early stage of stroke. The present study used a rat model of MCAO and PC12 cells (MCAO can mimic many of the salient features of the early stages of stroke) to further investigate the roles of miRNAs in neurite outgrowth associated with behavior. The three findings of our study were: i) the rat model of MCAO displayed significant behavioral deficits; ii) miR-125a expression was increased significantly in the rat model of MCAO, but Cadm2 expression was decreased; iii) Cadm2 (mRNA) is targeted by miR-125a. The results indicated that the disruption of neurite outgrowth via the miR-125a targeting of Cadm2 may play a role in the behavioral deficits associated with stroke and thus miR-125a may be a key target for drug development for the prevention of stroke.

An important finding of the present study was that miR-125a expression was increased significantly in the rats with MCAO. The microarray data and RT-qPCR data identified miR-125a to be upregulated in the rats with MCAO; these results are consistent with the hypothesis that some miRNAs can be differentially expressed in a stroke-affected brain. Previous studies have indicated that miR-125a is expressed or enriched in multiple myeloma $(39,40)$, psychiatric disorders (41) and cerebrovascular diseases (42). miR-125a has been shown to play a role in cancer development and metastasis, and to be involved in neurodegenerative diseases (43). However, little is understood about the roles of miR-125a in neurite outgrowth in stroke-afflicted rats. In our study, it was found that the upregulation of miR-125a was concomitant with the significant behavioral deficits displayed by the rats with MCAO. Therefore, the authors put forward the hypothesis that miR-125a may be associated with neurite outgrowth.

Previous studies have confirmed that several mRNAs are the direct targets of miR-125a, such as Gap43 and Cadm2. Among these target genes, Cadm2 plays an important role in neuronal growth, which regulates axon elongation and branching $(44,45)$. In the present study, the authors observed that expression of Cadm2 was decreased after overexpression of miR-125a and increased following miR-125a inhibition, paralleling with the neurite outgrowth. Bioinformatics analysis predicted that Cadm2 (mRNA) is targeted by miR-125a. Changes in expression of neural Cadm2 have been proposed to contribute to neurodevelopmental disorders in humans. The role of Cadm2 in neuronal differentiation remains, however, poorly understood (17). Therefore, the authors focused on the miR-125a to test the hypothesis. To confirm the hypothesis in vitro, a cortex neuronal $\mathrm{PC} 12$ cell line was used, which is an established cell model. PC12 cells were transfected with 
A

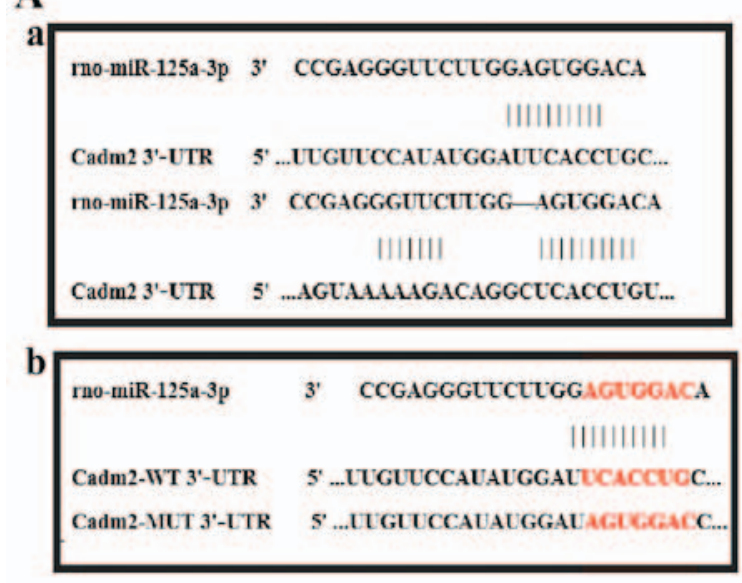

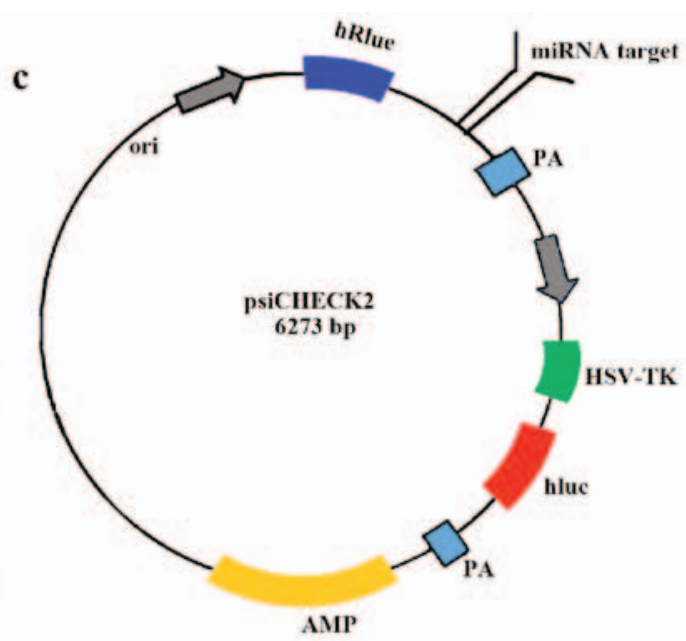

HSV-TK

B control mimic m-NC inhibitor $\mathrm{i}-\mathrm{NC}$
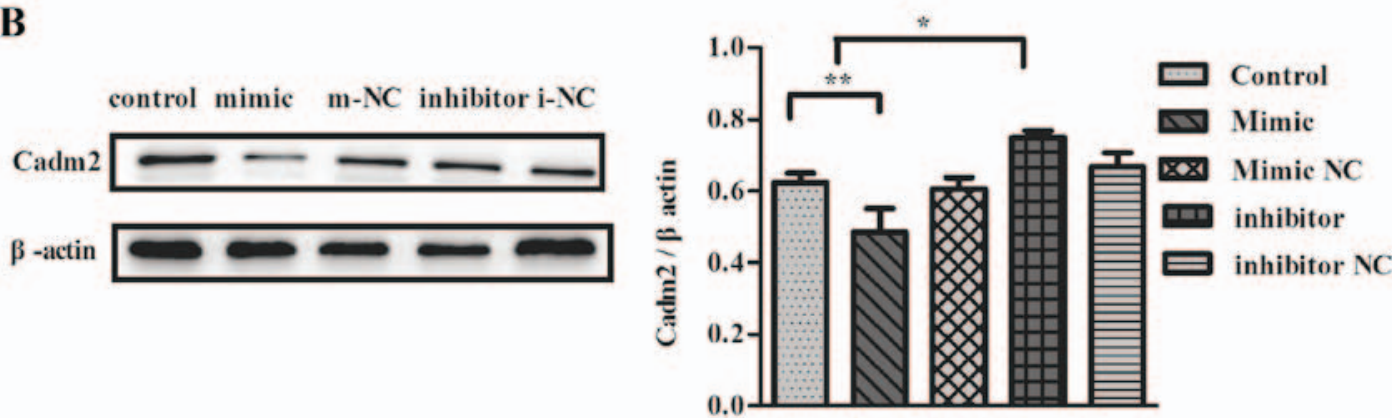

C

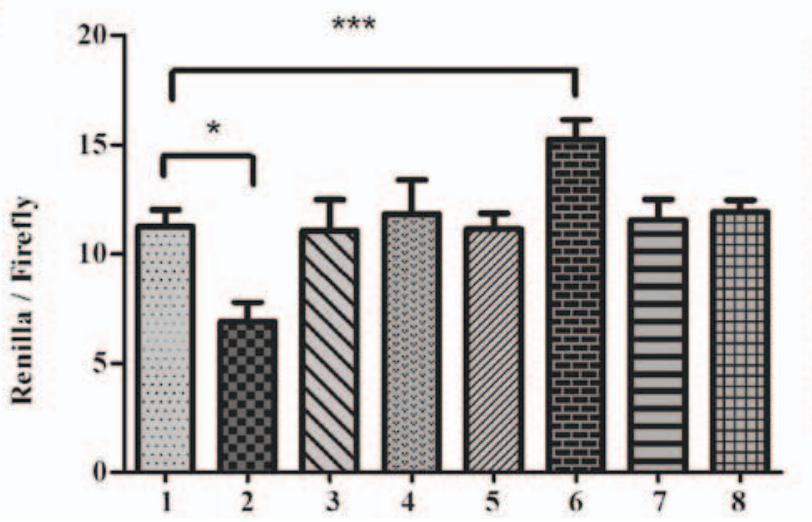

WT+miR-125 mimic

$\triangle$ MUT+mimic NC

MUT+miR-125 mimic

WT+inhibitor NC

WT+miR-125 inhibitor

MUT+inhibitor NC

표 MUT+miR-125 inhibitor

Figure 5. miR-125a regulates the expression of Cadm2. (A) (a) The target sites of miR-125a in the Cadm2 3'-UTR were predicted by bioinformatics software, TargetScan and miRNA.org. (b) The target sites of miR-125a in Cadm2-WT 3'-UTR and Cadm2-MUT 3'-UTR. (c) The construction profile of the psiCHECK2Cadm2 vector is presented in the diagram that contained the miR-125a-3p target sites in Cadm2 3'-UTR. (B) Expression of target protein Cadm2 in the PC12 cells were analyzed with the western blot assay, and the bands were quantified with ImageJ software. One-way analysis of variance was used to analyze the variances. By western blotting, the expression of the Cadm2 protein was efficiently decreased by miR-125a mimics, compared with the control group, and may be increased obviously when treated with miR-125a inhibitor. (C) The miR-125a mimic, inhibitor, mimic NC or inhibitor NC were co-transfected with Cadm2-WT 3'-UTR or Cadm2-MUT 3'-UTR into PC12 cells. miR-125a targeted the expression of Cadm2. One-way analysis of variance test was used to analyze the variances between groups. ${ }^{*} \mathrm{P}<0.05,{ }^{* * *} \mathrm{P}<0.01$ and ${ }^{* * * *} \mathrm{P}<0.001$ as indicated. miR, microRNA; UTR, untranslated region; WT, wild-type; NC, negative control; Cadm2, cell adhesion molecule 2.

miR-125a mimic or inhibitor, and then contrasted with the control group. The results of immunocytochemistry showed that mir-125 inhibited the expression of Cadm2 and that Cadm2 level was decreased in PC12 cells transfected with miR-125a mimics in western blotting and a luciferase reporter assay. These results suggested that Cadm2 is targeted by miR-125.

There are several important clinical implications in these results. First, stroke is one of the primary causes for morbidity and long-term disability worldwide. There is an urgent demand for sensitive and specific biomarkers for detection.
In the present study, the authors found that miR-125a was increased significantly in the cortex of model of MCAO. Thus, the miR-125a may be potential biomarkers for the early diagnosis of stroke. Secondly, currently there are no therapies to protect the progression of behavioral disturbances. The present studies have shown that miR-125a inhibited the neurite outgrowth in PC12 cells. Thirdly, the results support a new possible therapeutic target for stroke based on regulation of the miR-125-neurite outgrowth pathway on synaptic proteins. Inhibition of miR-125 appears a novel therapeutic option for behavioral disturbances in stroke. 
In conclusion, the observations suggest that the miR-125a is involved in behavioral disturbances in stroke and that inhibition of neurite outgrowth is due to the reduction of Cadm2 expression. The study suggested that miR-125a mediates neurite outgrowth by suppression of Cadm2, thereby inhibiting neurite outgrowth pathways in PC12 cells. This further indicated that altering the miR-125a-neurite outgrowth pathway may represent a targeted and mechanism-based therapeutic strategy against neurite outgrowth following stroke.

\section{Acknowledgements}

The present study was supported by the National Natural Science Foundation of China (grant nos. 81673770 and 81373525). Guangdong Provincial Natural Science Foundation of China (grant no. 2014A030313409).

\section{References}

1. Zhai WW, Sun L, Yu ZQ and Chen G: Hyperbaric oxygen therapy in experimental and clinical stroke. Med Gas Res 6: 111-118, 2016.

2. Boehme AK, Esenwa C and Elkind MS: Stroke risk factors, genetics, and prevention. Circ Res 120: 472-495, 2017.

3. Isabel C, Calvet D and Mas JL: Stroke prevention. Presse Med 45: e457-e471, 2016

4. Proietti M, Mairesse GH, Goethals P, Scavee C, Vijgen J, Blankoff I, Vandekerckhove Y and Lip GY; Belgian Heart Rhythm Week Investigators: Cerebrovascular disease, associated risk factors and antithrombotic therapy in a population screening cohort: Insights from the Belgian Heart Rhythm Week programme. Eur J Prev Cardiol 24: 328-334, 2017.

5. Perez-Alvarez MJ and Wandosell F: Stroke and neuroinflamation: role of sexual hormones. Curr Pharm Des 22: 1334-1349, 2016.

6. Gokce EC, Kahveci R, Gokce A, Sargon MF, Kisa U, Aksoy N, Cemil B and Erdogan B: Curcumin attenuates inflammation, oxidative stress, and ultrastructural damage induced by spinal cord ischemia-reperfusion injury in rats. J Stroke Cerebrovasc Dis 25: 1196-1207, 2016.

7. Gao L, Dong Q, Song Z, Shen F, Shi J and Li Y: NLRP3 inflammasome: A promising target in ischemic stroke. Inflamm Res 66: 17-24, 2017.

8. Wang J, Han D, Sun M and Feng J: Cerebral ischemic post conditioning induces autophagy inhibition and a HMGB1 secretion attenuation feedback loop to protect against ischemia reperfusion injury in an oxygen glucose deprivation cellular model. Mol Med Rep 14: 4162-4172, 2016.

9. Tiwari HS, Misra UK, Kalita J, Mishra A and Shukla S: Oxidative stress and glutamate excitotoxicity contribute to apoptosis in cerebral venous sinus thrombosis. Neurochem Int 100: 91-96, 2016

10. Wang X, Li H and Ding S: Pre-B-cell colony-enhancing factor protects against apoptotic neuronal death and mitochondrial damage in ischemia. Sci Rep 6: 32416, 2016.

11. Wu HJ, Wu C, Niu HJ, Wang K, Mo LJ, Shao AW, Dixon BJ, Zhang JM, Yang SX and Wang YR: Neuroprotective Mechanisms of Melatonin in Hemorrhagic Stroke. Cell Mol Neurobiol 37: 1173-1185, 2017.

12. Ballard C, Stephens S, Kenny R, Kalaria R, Tovee M and $\mathrm{O}^{\prime}$ Brien J: Profile of neuropsychological deficits in older stroke survivors without dementia. Dement Geriatr Cogn Disord 16 52-56, 2003.

13. Buesing C, Fisch G, O' Donnell M, Shahidi I, Thomas L, Mummidisetty CK, Williams KJ, Takahashi H, Rymer WZ and Jayaraman A: Effects of a wearable exoskeleton stride management assist system $\left(\mathrm{SMA}^{\circledR}\right)$ on spatiotemporal gait characteristics in individuals after stroke: A randomized controlled trial. J Neuroeng Rehabil 12: 69, 2015.

14. O' Hare A: Management of developmental speech and language disorders. Part 2: Acquired conditions. Arch Dis Child 101: 278-283, 2016

15. Sandig M, Rao Y and Siu CH: The homophilic binding site of the neural cell adhesion molecule NCAM is directly involved in promoting neurite outgrowth from cultured neural retinal cells. J Biol Chem 269: 14841-14848, 1994.
16. Kulahin $\mathrm{N}$ and Walmod PS: The neural cell adhesion molecule NCAM2/OCAM/RNCAM, a close relative to NCAM. Adv Exp Med Biol 663: 403-420, 2010.

17. Sheng L, Leshchyns'ka I and Sytnyk V: Neural cell adhesion molecule 2 promotes the formation of filopodia and neurite branching by inducing submembrane increases in $\mathrm{Ca}^{2+}$ levels. J Neurosci 35: 1739-1752, 2015.

18. Jeyaseelan K, Lim KY and Armugam A: MicroRNA expression in the blood and brain of rats subjected to transient focal ischemia by middle cerebral artery occlusion. Stroke 39: 959-966, 2008.

19. Wang Y and Cai Y: Obtaining human ischemic stroke gene expression biomarkers from animal models: A cross-species validation study. Sci Rep 6: 29693, 2016.

20. Zhou J and Zhang J: Identification of miRNA-21 and miRNA-24 in plasma as potential early stage markers of acute cerebral infarction. Mol Med Rep 10: 971-976, 2014

21. Brooks SA, Martin E, Smeester L, Grace MR, Boggess K and Fry RC: miRNAs as common regulators of the transforming growth factor (TGF)- $\beta$ pathway in the preeclamptic placenta and cadmium-treated trophoblasts: Links between the environment, the epigenome and preeclampsia. Food Chem Toxicol 98: 50-57, 2016.

22. Su SH, Wu CH, Chiu YL, Chang SJ, Lo HH, Liao KH, Tsai CF, Tsai TN, Lin CH, Cheng SM, et al: Dysregulation of vascular endothelial growth factor receptor- 2 by Mmultiple miRNAs in endothelial colony-forming cells of coronary artery disease. J Vasc Res 54: 22-32, 2017.

23. Longqiu Y, Pengcheng L, Xuejie F and Peng Z: A miRNAs panel promotes the proliferation and invasion of colorectal cancer cells by targeting GABBR1. Cancer Med 5: 2022-2031, 2016.

24. Tang W, Cai P, Huo W, Li H, Tang J, Zhu D, Xie H, Chen P, Hang B, Wang S, et al: Suppressive action of miRNAs to ARP $2 / 3$ complex reduces cell migration and proliferation via RAC isoforms in Hirschsprung disease. J Cell Mol Med 20: 1266-1275, 2016.

25. Quan L, Wang Y, Liang J, Qiu T, Wang H, Zhang Y, Zhang Y, Hui Q and Tao K: Screening for genes, transcription factors and miRNAs associated with the myogenic and osteogenic differentiation of human adipose tissue-derived stem cells. Int J Mol Med 38: 1839-1849, 2016.

26. Filip AA, Grenda A, Popek S, Koczkodaj D, MichalakWojnowska M, Budzyński M, Wąsik-Szczepanek E, Zmorzyński S, Karczmarczyk A and Giannopoulos K: Expression of circulating miRNAs associated with lymphocyte differentiation and activation in CLL-another piece in the puzzle. Ann Hematol 96: 33-50, 2017.

27. Bao W, Greenwold MJ and Sawyer RH: Expressed miRNAs target feather related mRNAs involved in cell signaling, cell adhesion and structure during chicken epidermal development. Gene 591: 393-402, 2016.

28. Martinez-Sanchez A, Rutter GA and Latreille M: MiRNAs in $\beta$-cell development, identity, and disease. Front Genet 7: 226, 2017.

29. Coutinho-Camillo CM, Lourenço SV, de Araújo Lima L, Kowalski LP and Soares FA: Expression of apoptosis-regulating miRNAs and target mRNAs in oral squamous cell carcinoma. Cancer Genet 208: 382-389, 2015.

30. Venkatadri R, Muni T, Iyer AK, Yakisich JS and Azad N: Role of apoptosis-related miRNAs in resveratrol-induced breast cancer cell death. Cell Death Dis 7: e2104, 2016.

31. Privitera AP, Distefano R, Wefer HA, Ferro A, Pulvirenti A and Giugno R: OCDB: A database collecting genes, miRNAs and drugs for obsessive-compulsive disorder. Database (Oxford) 2015: bav069, 2015.

32. Dajas-Bailador F, Bonev B, Garcez P, Stanley P, Guillemot F and Papalopulu N: microRNA-9 regulates axon extension and branching by targeting Map1b in mouse cortical neurons. Nat Neurosci 15: 697-699, 2012.

33. Lu XC, Zheng JY, Tang LJ, Huang BS, Li K, Tao Y, Yu W, Zhu RL, Li S and Li LX: MiR-133b Promotes neurite outgrowth by targeting RhoA expression. Cell Physiol Biochem 35: 246-258, 2015.

34. Longa EZ, Weinstein PR, Carlson S and Cummins R: Reversible middle cerebral artery occlusion without craniectomy in rats. Stroke 20: 84-91, 1989.

35. Zeng L, Liu J, Wang Y, Wang L, Weng S, Tang Y, Zheng C, Cheng Q, Chen S and Yang GY: MicroRNA-210 as a novel blood biomarker in acute cerebral ischemia. Front Biosci (Elite Ed) 3: 1265-1272, 2011.

36. Xu W, Jin W, Zhang X, Chen J and Ren C: Remote limb preconditioning generates a neuroprotective effect by modulating the extrinsic apoptotic pathway and TRAIL-receptors expression. Cell Mol Neurobiol 37: 169-182, 2017. 
37. Chi W, Meng F, Li Y, Li P, Wang G, Cheng H, Han S and Li J: Impact of microRNA-134 on neural cell survival against ischemic injury in primary cultured neuronal cells and mouse brain with ischemic stroke by targeting HSPA12B. Brain Res 1592: 22-33, 2014.

38. Mercado AT, Yeh JM, Chin TY, Chen WS, Chen-Yang YW and Chen CY: The effect of chemically modified electrospun silica nanofiber on the mRNA and miRNA expression profile of neural stem cell differentiation. J Biomed Mater Res A 104: 2730-2743, 2016.

39. Bi C, Chung TH, Huang G, Zhou J, Yan J, Ahmann GJ, Fonseca R and Chng WJ: Genome-wide pharmacologic unmasking identifies tumor suppressive microRNAs in multiple myeloma. Oncotarget 6: 26508-26518, 2015.

40. Alzrigat M, Parraga AA, Agarwal P, Zureigat H, Österborg A, Nahi H, Ma A, Jin J, Nilsson K, Öberg F, et al: EZH2 inhibition in multiple myeloma downregulates myeloma associated oncogenes and upregulates microRNAs with potential tumor suppressor functions. Oncotarget 8: 10213-10224, 2017.

41. Camkurt MA, Karababa F, Erdal ME, Bayazit H, Kandemir SB Ay ME, Kandemir H, Ay Öİ, Çiçek E, Selek S, et al: Investigation of dysregulation of several MicroRNAs in peripheral blood of schizophrenia patients. Clin Psychopharmacol Neurosci 14: 256-260, 2016
42. Dai D, Lu Q, Huang Q, Yang P, Hong B, Xu Y, Zhao W, Liu J and Li Q: Serum miRNA signature in Moyamoya disease. PLoS One 9: e102382, 2014

43. Dasdag S, Akdag MZ, Erdal ME, Erdal N, Ay OI, Ay ME, Yilmaz SG, Tasdelen B and Yegin K: Effects of $2.4 \mathrm{GHz}$ radiofrequency radiation emitted from Wi-Fi equipment on microRNA expression in brain tissue. Int J Radiat Biol 91: 555-561, 2015

44. Futerman $\mathrm{AH}$ and Banker GA: The economics of neurite outgrowth - the addition of new membrane to growing axons. Trends Neurosci 19: 144-149, 1996.

45. Blackmore MG, Moore DL, Smith RP, Goldberg JL, Bixby JL and Lemmon VP: High content screening of cortical neurons identifies novel regulators of axon growth. Mol Cell Neurosci 44: 43-54, 2010.

This work is licensed under a Creative Commons Attribution-NonCommercial-NoDerivatives 4.0 International (CC BY-NC-ND 4.0) License. 